\title{
Operational Performance of MOSFIRE with Its Cryogenic Configurable Slitmask Unit at the W. M. Keck Observatory
}

\author{
Marc Kassis*a $^{*}$, Ian S. McLean ${ }^{\text {b }}$, Charles C. Steidel ${ }^{\mathrm{c}}$, Keith Matthews ${ }^{\mathrm{c}}$, James E. Lyke ${ }^{\mathrm{a}}$, Luca Rizzi ${ }^{\mathrm{a}}$, \\ Dwight Chan ${ }^{\mathrm{a}}$, Jason Weiss ${ }^{\mathrm{b}}$, Sean Adkins ${ }^{\mathrm{a}}$, Peter Spanoudakis ${ }^{\mathrm{d}}$, Philippe Schwab ${ }^{\mathrm{d}}$, Emmanuel \\ Onillion $^{\mathrm{d}}$, Leszek Lisowski ${ }^{\mathrm{d}}$, Patrick Theurillat ${ }^{\mathrm{d}}$, Olivier Chételat ${ }^{\mathrm{d}}$ \& Christophe Meier $^{\mathrm{d}}$ \\ ${ }^{\mathrm{a}}$ W. M. Keck Observatory, 65-1120 Mamalahoa Hwy, Kamuela, HI, USA, 96743; \\ ${ }^{\mathrm{b}}$ University of California, Los Angeles, CA, USA 90095-1547; \\ ${ }^{\mathrm{c}}$ California Institute of Technology, Pasadena, CA, USA, 91125; \\ ${ }^{d}$ Centre Suisse d'Electronique et de Microtechnique, Jaquet-Droz 1, CH-2007 Neuchâtel, Switzerland
}

\begin{abstract}
The Multi-Object Spectrograph for Infrared Exploration (MOSFIRE) achieved first light on the W. M. Keck Observatory's Keck I telescope on 4 April 2012 and quickly became the most popular Keck I instrument. One of the primary reasons for the instrument's popularity is that it uses a configurable slitmask unit developed by the Centre Suisse d'Electronique et Microtechnique (CSEM SA) to isolate the light from up to 46 objects simultaneously. In collaboration with the instrument development team and CSEM engineers, the Keck observatory staff present how MOSFIRE is successfully used, and we identify what contributed to routine and trouble free nighttime operations.
\end{abstract}

Keywords: spectrometer, near-infrared, multi-object, Configurable Slit Unit, MOSFIRE, W. M. Keck Observatory, efficient night operations

\section{INTRODUCTION}

MOSFIRE is the multi-object spectrograph and wide-field infrared camera at the W. M. Keck Observatory. This large vacuum-cryogenic instrument is located in a rotating module at the Keck I Cassegrain focus. The resolving power of MOSFIRE for a 0.7 " ( $\sim 3$ pixels) slit on the sky is R 3500, and spectroscopy can be performed in the $\mathrm{Y}, \mathrm{J}, \mathrm{H}$ or $\mathrm{K}$ bands. A unique feature of MOSFIRE is the cryogenic Configurable Slit Unit (CSU) developed in collaboration with the Swiss Center for Electronics and Microtechnology (CSEM) ${ }^{1}$. The CSU allows observers to create slit masks over the $6.1^{\prime} \times 6.1^{\prime}$ field of view (FOV) using 46 pairs of bars that move in from each side. Each bar can traverse the entire $267 \mathrm{~mm}$ field of view, and each bar carries a precision knife-edge that acts as one half of a slit. The standard slit length associated with each bar is 7.1", but bars can be stacked to produce longer slits up to the full extent of the field. Boxes used for alignment stars during set-up can be reconfigured on the fly back to narrow slits for science targets, and the entire pattern of slits can be reconfigured under computer control in less than six minutes. No thermal cycling of the large vacuum chamber is needed. When the bars are fully retracted, and the diffraction grating is switched to a mirror, MOSFIRE becomes a widefield imager. Other features of MOSFIRE include a separate offset guider and calibration unit, a heated double entrance window to prevent condensation, a tip-tilt flexure compensation system, a double filter wheel, a rotating Lyot stop, and a grating turret with two fixed stops for repeatable positioning. The detector is a Teledyne $2 \mathrm{~K}$ x $2 \mathrm{~K}$ H2-RG with a cutoff wavelength of 2.5 microns, which is operated by a SIDECAR ASIC.

MOSFIRE was developed by teams from the University of California, Los Angeles (UCLA), the California Institute of Technology (CIT) and the University of California, Santa Cruz, (UCSC). The Co-Principal Investigators were Ian S. McLean (UCLA) and Charles C. Steidel (CIT), and the optical design was led by Harland Epps (UCSC). In sheer size, mass and complexity, MOSFIRE was one of the most challenging cryogenic infrared spectrographs ever attempted. After more than seven years in development, MOSFIRE was delivered to the Keck Observatory on February 8, 2012 and "first light" on the Keck 1 telescope was obtained on April 4, 2012. Requirements and design details for MOSFIRE were described in McLean et al. (2010) $)^{2}$, and the performance of the detector and the as-built instrument, based on early commissioning results was presented in Kulas, McLean, and Steidel (2012) ${ }^{3}$ and McLean et al. (2012) ${ }^{4}$. These papers

Ground-based and Airborne Instrumentation for Astronomy VI, edited by Christopher J. Evans, Luc Simard, Hideki Takami Proc. of SPIE Vol. 9908, 99080E · (c) 2016 SPIE · CCC code: 0277-786X/16/\$18 · doi: 10.1117/12.2233006 
also addressed risk management, new fabrication techniques, and software development. Measurements on the telescope have confirmed laboratory predictions that MOSFIRE is a high-throughput and sensitive spectrograph and imager.

In the four years since commissioning, MOSFIRE has been highly productive and in great demand. MOSFIRE is the most frequently used instrument on the Keck I telescope with an average 64 nights scheduled per semester. Every semester, the productivity of MOSFIRE has increased as a direct result of steady improvement efforts completed collaboratively by Keck Observatory staff, MOSFIRE development team members, and CSEM engineers. The observing community benefits directly from the improvement efforts by taking advantage of a $15 \%$ increase in science time since commissioning (see Figure 1). MOSFIRE's productivity has resulted in 54 research papers through the end of 2015. A comparison of the publication record during the first few years following commissioning Keck Observatory's three multi-object spectrographs is also presented in Figure 1, which shows that MOSFIRE is keeping pace with two very successful optical instruments. When compared to Keck Observatory's average publication record over all instruments, MOSFIRE is leading the way in its first years of operation.

Of course, most of the published MOSFIRE science involves the high-redshift universe. MOSFIRE has enabled several large galaxy surveys such as $\mathrm{KBSS}^{5}, \mathrm{MOSDEF}^{6,7}$ and $\mathrm{TKRS}^{8}$, but remarkable results have also been obtained on the transmission spectrum of an exoplanet ${ }^{9}$. MOSFIRE has also contributed to several record-breaking galaxy redshifts beyond $\mathrm{z}=7.5$, thanks to the instrument's sensitivity and stability ${ }^{10}$.
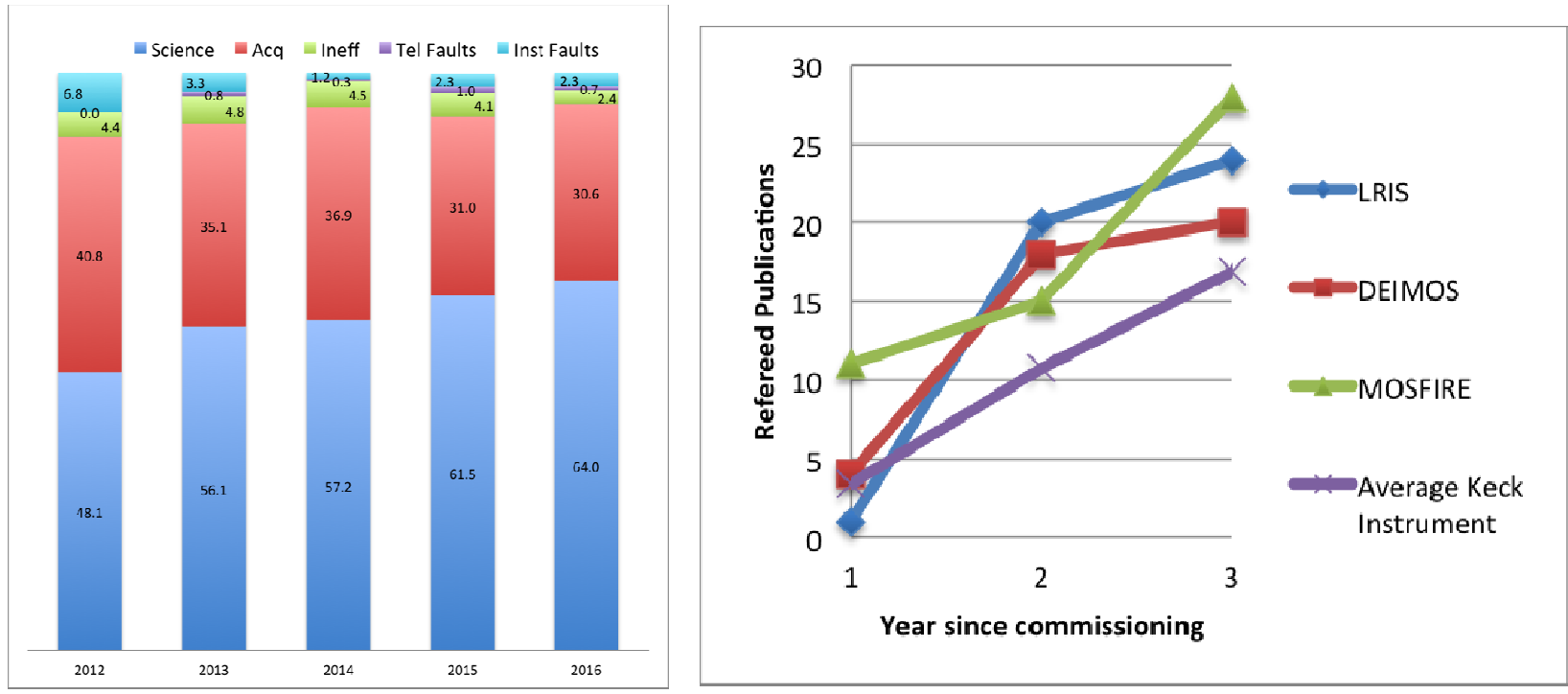

Figure 1. Annual nightly average of the fraction of time spent collecting science data compared to other activities (left); the number of refereed publications since commissioning for the Keck Observatory's three multi-object spectrographs: DEIMOS $^{11}$, LRIS ${ }^{12,13}$, and MOSFIRE (right).

The instrument development team faced tight constraints in what was intended as a design-to-cost project. Funding for MOSFIRE was provided through a private-public partnership involving Gordon and Betty Moore and the National Science Foundation. At the start of the project in 2005, four high-risk areas were identified: the cryogenic configurable slit unit (CSU); the large cryogenic optics operating at $120 \mathrm{~K}$; mechanical flexure of MOSFIRE at the Cassegrain focus; detector performance using the SIDECAR ASIC technology. It was also clear that user-friendly software would be essential in order to make it easy to use MOSFIRE. Significant effort was expended to test the entire instrument in the lab under conditions similar to those on the telescope, including variable elevation and axial rotation settings. Of course, conditions in the real observatory environment can be more challenging. In the end, the development team delivered a working instrument that produced outstanding science from first light. In this paper, we discuss lessons learned over four years of routine, and increasingly efficient, night-time use of MOSFIRE from the viewpoint of observatory instrument support and operations. As already mentioned, MOSFIRE's spectroscopic multi-object capability is made possible by the CSU, thus significant emphasis is placed on operational issues associated with this remarkable mechanism in the sections to follow. Orchestrated MOSFIRE observing sequences are eased by several well thought out software design choices, all of which help both observers and support astronomers to control MOSFIRE efficiently, and quickly learn how to use the instrument. We are also continuing to pursue new modes, and explore solutions to existing challenges, 
and therefore, in the second-to-last section we discuss future activities. Finally, we conclude with a brief summary of the science enabled by MOSFIRE.

\section{USER INTERFACE AND SOFTWARE}

For observers, and even for new support astronomers, learning to operate and troubleshoot MOSFIRE is straight forward. The learning curve is reduced because the user interface is based on heritage code developed for other Keck instruments, and features both a graphical user interface (GUI) and a command-line interface (CLI) that may be used to script instrument actions. All instrument GUIs and scripts are designed to interface with a keyword library where setting a keyword=value pair initiates an instrument function such as moving a filter wheel or retrieving the current detector temperature. In addition to modifying single keyword-value pairs, the GUIs also call more advanced Cshell and python scripts that coordinate many keyword modifications, and also check for status execution when complex sequences are needed. The flexibility available in scripting keywords, and the ease with which the Java code can be updated, helps support staff more easily accommodate observer requests for improvements and modifications.

Although MOSFIRE is probably the most sensitive near-infrared imager on the planet, astronomers use imaging mode to collect only $4.7 \%$ of MOSFIRE science data, as determined by the number of FITS files in the Keck Observatory Archive (KOA). Thus far, the medium band imaging filters (J2, J3, H1, and H2) are rarely used, accounting for less than $0.5 \%$ of the total science data collected. On the other hand, K-band imaging and spectroscopic data comprise almost half the observations obtained with MOSFIRE (see Figure 2). The medium band J2 filter covers a slightly shorter wavelength region than the broad-band $\mathrm{J}$ filter, and a few observing teams have taken advantage of the $\mathrm{J} 2$ filter's shorter wavelength coverage in spectroscopic mode $(0.3 \%)$. Because observing teams predominantly desire the spectroscopic multiplexing capabilities of MOSFIRE, post-delivery enhancements have emphasized improving spectroscopic efficiencies.

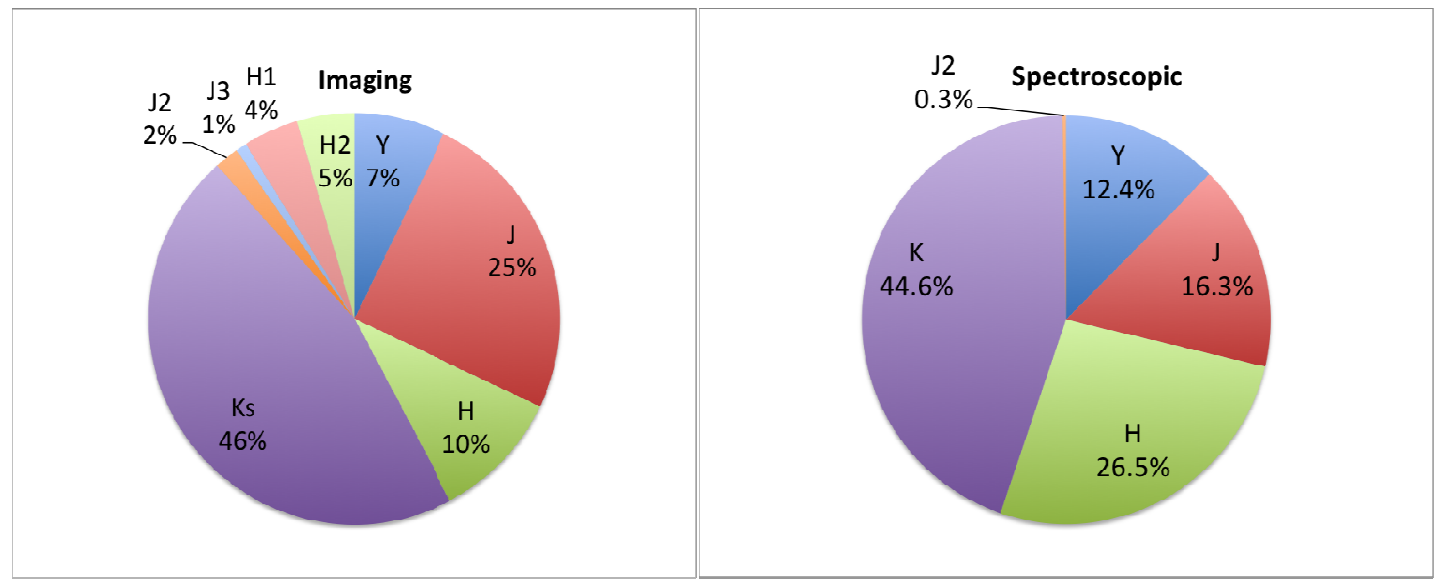

Figure 2. Fractional use for filters in imaging (left) and spectroscopic (right) modes. Spectroscopy observations are $95.3 \%$ of the total data acquired to date, while imaging mode is used to acquire $4.7 \%$ of science data.

Below we list the operational benefits, and some limitations, of the software when MOSFIRE was commissioned, and describe improvements driven by on-sky experience and observer requests. Overall, the MOSFIRE software has been exceptionally good and stable.

- MAGMA is the GUI used for both pre-run mask design and real-time control of the CSU at the observatory. Because observers use the stand-alone version for mask design, the observers are already familiar with one of the real-time GUIs, thus reducing the learning curve at the telescope.

- Mask execution is a two-step process: setup and execution. The two-step process almost eliminates observers from configuring the wrong mask, and because configuration times are of order minutes, this speed bump saves time on sky. The software leads observers through the two-step process by highlighting MAGMA GUI buttons in green, which helps the novice observer.

- Because masks may be designed or modified in real time, observers can use MAGMA to adjust masks to seeing conditions by changing the slit width, or create new masks during the night to pursue new or modified science goals. 
- The MOSFIRE Desktop is a Java platform that contains all status and control GUIs for the instrument, with the exception of MAGMA. This very integrated design ensures that there is never a doubt in the observer's mind whether all the software is running or not, and it also keeps the local display desktop clean and well-organized. In addition, the desktop layout of the sub GUIs is easily reconfigured using layout specification files.

- Coordinated instrument moves are completed with just one click on the "observing mode" sub GUI on the MOSFIRE desktop. With one click, observers are able to configure the grating, filter, and pupil, and this saves time at night.

- Status GUIs for motion control provide clear indications for each mechanism and for exposure control.

- Errors in motions are clearly displayed.

- The exposure control sub GUI within the MOSFIRE desktop provides an excellent estimate of the "time to complete" all observations, including overheads, which were updated after the first observing semester. The exposure status GUI does a wonderful job of indicating the progress of a current exposure sequence via progress meters for the current exposure, dither pattern, and the number of repeats.

- Functions for aborting at any time during an exposure sequence are robust and may be used to time aborts when exposures are complete.

- The MOSFIRE code re-uses software common to all Keck multi-object spectrographs for slitmask alignment, making it much easier to cross train observers on multiple instruments.

- The utility DS9 is used for real time image display. This is beneficial because the observatory's other multiobject spectrographs both use DS9 for data display; again, cross-training on these instrument is improved. MOSFIRE uses DS9 to overlay bar positions in imaging mode and automatically displays three frames: a raw image, a differenced A-B subtraction image for all observing modes, and an optional third frame for a mosaic image when multiple pointings are used during dithering in imaging mode. The overlays, and the other two frames, are critical tools in assessing CSU bar positions and have been essential tools at night for quick looks at the data as well as troubleshooting.

- The MOSFIRE team included a very narrow band filter originally intended for imaging science. This filter has turned out to be useful for aligning bright stars because it minimizes flux and mitigates charge persistence from over illumination. We typically use this filter when aligning calibration stars of $7-9^{\text {th }}$ magnitude on a long slit.

Although there were very few deficiencies in the code on delivery, afternoon and on-sky experience was essential to fine-tune the software. Below, we list the most prominent aspects of the user interfaces that were improved to reduce lost time on the sky, and ease minor observer frustrations. Again the flexibility built into the GUI code as well as the ability to script complex sequences using keywords was instrumental in quick software improvements.

- MOSFIRE was delivered with a calibration GUI and scripts designed to automate acquisitions of arcs and flats for a set of CSU mask configurations. Automating a set of calibrations frees observers and reduces mistakes, and this is a common tool made available for most Keck instruments. As observers started calibrating multiple masks daily, the Keck staff learned how to make the software bullet-proof, and after the first semester of operation, the code was re-written to make it more robust and to take advantage of a an improved dome lamp illumination system installed a year later that was designed with MOSFIRE spectroscopic flats in mind.

- The grating, filter wheels, and pupil stop are controlled via a coordinated keyword that initiates motion for those three mechanisms. Over time the, support staff wrote and updated scripts to handle properly all possible status events reported by the mechanism servers in order to assess when all motions were complete and the mechanisms were in position. It required two years of on-sky experience to develop the mechanism control scripts fully, in part because different status outputs kept being discovered. Good status-handling is an essential component for many scripts, including the calibration software, and it has been very robust despite discovering an additional transitional status this past April.

- An umbrella keyword server, designated as the global server, was implemented as the point of contact between all clients (keyword calls and GUIs). Sub-servers that feed information to the global server functioned well on delivery, but it took a year to troubleshoot and improve the global server itself to make it as robust as possible for routine night operations at the telescope. The global server could block information flow and would occasionally fill up its message queue. Although the time to recover from these errors was relatively small $(<5$ $\mathrm{min}$ ), the frequency of problems in the first year demanded that we solve this problem, and we have.

After commissioning, the observing community quickly challenged both the Keck support staff and the MOSFIRE team to create additional tools that improved on-sky efficiencies, and better accommodated their science programs. Again, the 
flexibility of the software helped Keck staff to modify or create new code to meet observer needs on very short time scales. Below, we highlight some of the major adjustments:

- We created a two-position longslit and scripted an observing sequence to acquire calibrations specifically in $\mathrm{H}$ and $\mathrm{K}$ bands in order to ensure full wavelength coverage. For a single slit position, one cannot obtain full wavelength coverage in $\mathrm{H}$ or $\mathrm{K}$, so two positions are needed to obtain the short and long wavelength spectral coverage for those filters. Spectroscopic flux calibrators in particular are acquired using this technique.

- We developed sequencing GUIs and scripts to acquire data automatically in multiple spectroscopic filters.

- For multiple masks in dense fields, we created tools to optimize telescope pointing. Because the telescope pointing is very accurate for small slews within the guider FOV, and because the CSU can position slits very precisely, we save several minutes by avoiding mask alignments by instead simply offsetting the telescope between mask positions.

- We developed single object alignment software for relatively bright sources $(7<\mathrm{J}$ mag $<13)$ that used the narrow band filter mentioned above. This software was folded into our slitmask alignment software so observers have one-stop-shopping for all target coarse and fine tune positioning.

- We added more filter pair combinations that result in a "dark" filter because the transmission of the two filters does not overlap. These combinations minimize filter moves and again increase observing efficiencies.

- Exoplanet observations required aligning objects in $10^{\prime \prime}$ wide longslits, so we developed longslit alignment software for two objects when alignment boxes were not used.

- The J2 filter reaches a slightly shorter wavelength than the broad-band J filter, and some observers are using the J2 filter to meet their spectroscopic needs. We updated scripts for calibrations and data acquisition to provide observers with this option.

\section{CRYOGENIC SLITMASK USAGE}

Reconfiguring the CSU during the night is an orchestrated process with well-documented procedures that observers are strongly encouraged to follow when switching targets. These procedures are designed to avoid two things: over exposing the detector to light, and moving the CSU bars when the bars are parallel to the horizon (see section 4.1 for a discussion of horizontal bar movement). Over exposing the H2RG detector leads to charge persistence ${ }^{3}$ that will contaminate science observations. Thus, before slewing or reconfiguring the CSU, observers are instructed to install a dark filter. Again, with observing experience, the original software was modified to help streamline our observing procedures.

To move the CSU, the first step is to select a prepared mask design and then upload the bar positions to the CSU controller. Uploading the bar position is a serial process; when each bar position is loaded, the CSU status GUI indicates that a change is needed by updating the status to "setup." The second step is to execute the mask. The two-step process is beneficial because it gives observers a chance to double check that the desired mask is setup and ready to be executed.

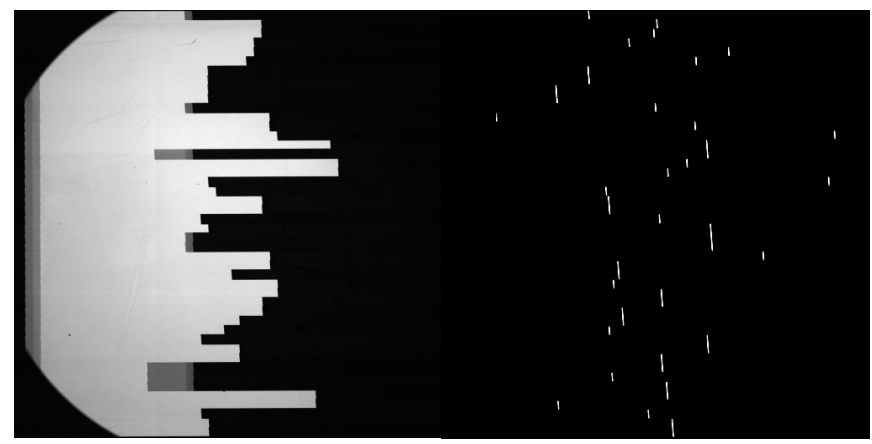

Video 3. CSU_open_mask (left) and CSU_mask_change (right) are two videos that show common mask reconfigurations. The open mask shows the bar movements from an open configuration that was used for imaging to a mask configuration. The mask change video shows the bar motions when observers switch from one mask to a second mask.

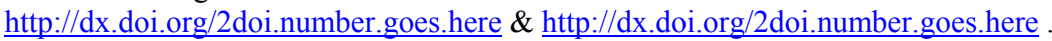

Two videos (Video 3) show the typical motions for the CSU as it is used in MOSFIRE. The left video starts with all of the bars retracted or in the "open" configuration; this is the configuration used for imaging. The images for the movie were acquired using thermal radiation from the back of the dust cover at the front of the instrument; the dust cover is at 
ambient dome temperatures. The corners appear dark in imaging mode because there is a field stop in the focal plane; the full array is used in spectroscopic mode.

From the open position, bars move efficiently by first engaging a ratchet for all bars on the right hand side of the FOV and then releasing the brakes for all bars simultaneously. The bars then move one coarse step (four teeth pitch) and then the brakes engage and the ratchets release to move back one coarse step. This cyclic process repeats itself until one or more of the bars are within one coarse step of their final position, at which point, the bars are dropped from the coarse positioning sequence. Coarse positioning continues for right hand side bars until all bars are near their final destination. The coarse positioning is then completed for the left hand set of bars. For fine positioning, the software moves bars serially to slowly move the bars to their final destination. Again, all fine motion is completed for the right hand bars first, and then the process repeats for the left hand set. Moving from an "open" configuration to a user-designed slit mask configuration takes approximately $4.5 \mathrm{~min}$. For a more complete description of the CSU see Spanoudakis et al. (2008) ${ }^{1}$.

The second movie (right) captures a sequence of bar motions when going from a science mask with $0.7^{\prime \prime}$ wide slits to an alignment mask for a new target field; this configuration change is more typical during night time observing. For the alignment mask, most slits are positioned with widths of $0.7^{\prime \prime}$ but five of the slits are configured as $4.0^{\prime \prime}$ wide alignment boxes. The motion of the bars in the second video is running at twice normal speed. First, bars are fine positioned to the nearest coarse step that will enlarge the slit width. Depending on the current position of the bars and how bars may be grouped in subsets, the initial fine motion starts with either the left or right bar in a slit pair. In the video, most of the top third of the slits open to the right initially while the slits in the middle of the mask open to the left. Then the other side of the slit pairs are fine tuned to the nearest coarse step position. In some cases, this has the effect of closing off a slit, while in others the slit opens a little more. Then, subsets of bars are coarse positioned. For a slit pair, the bar nearest to the final location is positioned first. If the new location of a slit pair is to the left of the current location, the left bar undergoes coarse and fine positioning before the right bar. The process of reconfiguring all bars from a new mask requires about 4.5 minutes.

Between September 2012 (start of regular science operations) and April 2016 (anniversary of first light), 7,374 different mask configurations were completed as recorded by the FITS header information. Out of those 7,374 masks, most are either multi-object science masks or the alignment mask that is paired with it $(2,845 \times 2)$. Other masks configurations are used for focusing the telescope, imaging the field, acquiring standards, or initializing the mechanism. On consecutive nights, the same mask may be configured for science use, but because bar positioning is so repeatable, calibration files are only acquired once per run, reducing the number of needed configurations. Our estimate for the lifetime of the mechanism was 10 years with an estimate of 2400 masks configured annually. At an average mask configuration rate of 2011 masks per year, we should meet and exceed our 10 year goal.

Although different observing teams design multi-object slitmasks that are unique to their own scientific programs, there are a handful of common mask designs that observers can use. The common masks use only a subset of bars in the central region of the mask as shown in Figure 4. The left most image of Figure 4 shows the central region of an observer designed mask. Figure $4 \mathrm{~b}$ then shows the central region opened for imaging use when focusing the telescope. To save reconfiguration time, we efficiently reposition only the bars that are blocking the central $50^{\prime \prime}$ FOV. Thus, instead of a rigid square configuration, the imaging area has sections where either the left or right bar in a pair remains in position for the science slits while the other in the pair is moved to the open focus position. The mask shown in Figure 4c is used for single objects, often used for standard stars, and is a "short" longslit. Lonslits may be as long as the full FOV (6') but again, to save reconfiguration time; typically only five slits are configured for a longslit. Note that the software closes alignment boxes in longslit mode to ensure those regions of the detector are not overexposed. The right most image of Figure 4 shows a "long2pos" mask that is a two-position, three-bar length longslit. The central bar is an alignment box just like the typical longslit.

Observers typically design masks with targets spread over a $3^{\prime}$ x $6^{\prime}$ FOV which results in different wavelength coverage of the $\mathrm{H}$ and $\mathrm{K}$ bands depending on slit location. The long2pos mask and the observing software that goes with it, was developed in the second year of science operations to help observers efficiently acquire calibration spectra for the full $\mathrm{H}$ and $\mathrm{K}$ bands. The flexibility of the software and the CSU was leveraged to create these additional observing tools. Because of the large offsets needed to put a standard star at both long2pos positions, a guide star must be near the central region of the guider. However, we often complete the quick set of standard observations unguided because exposure times for standards are relatively short (4-10 seconds). 

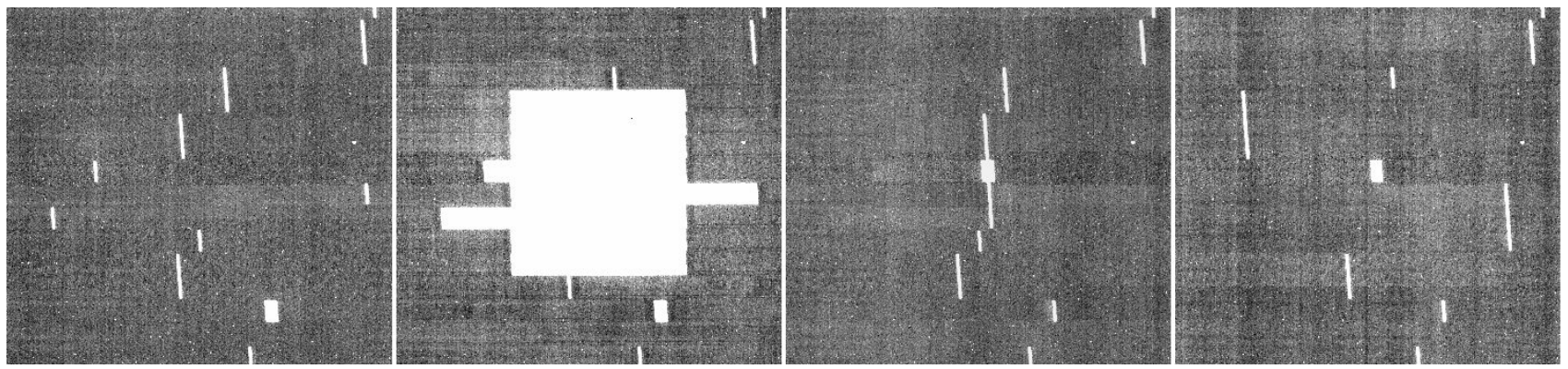

Figure 4. Images of the central portion of the MOSIFIRE FOV that show four types of common mask configurations consisting of 16 bar pairs. From left to right the images show a) a multi-object slitmask, b) an open configuration used for focusing, c) a single slit with a length of five bars, and d) a longslit two position mask. Note that the very top and the very bottom slits of all images have the same slit positioning.

Because the central region is reconfigured more frequently, both the number of individual bar configurations and the total distance traveled by those bars are larger than the median value, as can be seen in Figure 5. Bars 45 and 46 (peak in bar moves) are commanded to move the most because this set of bars is used for aligning targets on a longslit mask before being reconfigured to a standard slit of width of $0.7^{\prime \prime}$. There is a small decrease in the number of moves and distance traveled for the lowest and highest numbered bars. These bars move in regions of the FOV where there is less access to the full field (see Video 3), and therefore, the distance traveled will predominantly be within the FOV. Overall, the bars have moved between 200 and 240 meters

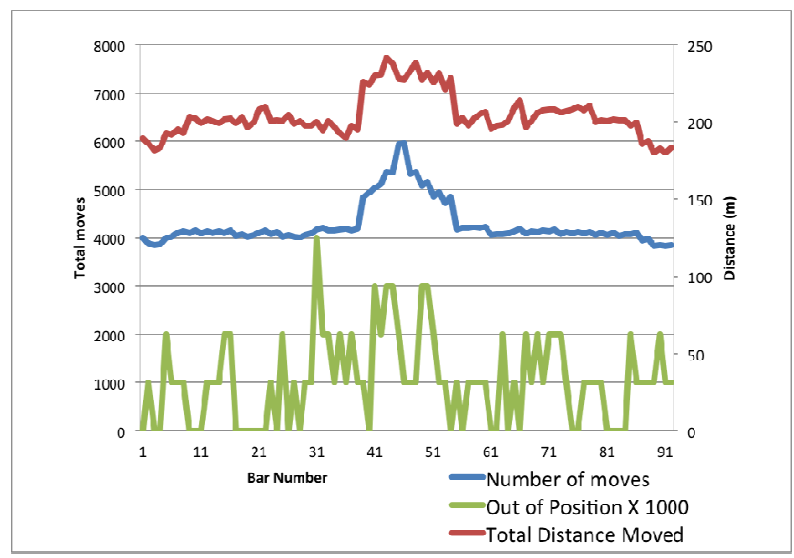

Figure 5. The total number of moves completed between Aug 2012 and April 2016, the total distance traveled by each bar, and the number of times each bar was detected to be out of position (x1000).

All of the Keck observatory multi-object spectrographs use the same software to align the slitmasks on target. This software is called the Slitmask Alignment Tool (SAT) ${ }^{14}$, and the observers are trained to use this software at night. Because the CSU bars have discrete slit lengths and are slanted by roughly four degrees relative to the detector rows and columns, the SAT needed three prominent modifications for use with MOSFIRE.

- Instead of centering stars in alignment boxes $4 "$ x $4 "$ wide as is the case for the milled slitmaks used by LRIS and DEIMOS, the alignment software was modified to center stars at an expected location in an elongated box. MAGMA stores the alignment star positions in FITS extensions that are saved with every data set.

- The box profiles are different because the boxes are slanted relative to the detector rows and columns to improve sampling, and this therefore results in a 4 " x 7 "parallelogram on the detector. The box profiles were updated to account for the unique shape.

- We also implemented sky subtraction data acquisition and reduction. The infrared sky is sometimes the dominant source of emission in the alignment box, swamping the alignment star flux. A sky image acquired 10" east of the target at the start of an alignment sequence is subtracted from the target image, which not only removes the sky emission but also minimizes pixel-to-pixel variations similar to a flat, thereby reducing noise that confuses fainter targets. 
Figure 6 shows how the software uses the images and FITS header information to find and align a star in a box. The software measures the peak right of the desired location (red line) which is offset from the box center. After aligning the star in the box, observers then redeploy the box to a slit to maximize their science observations.
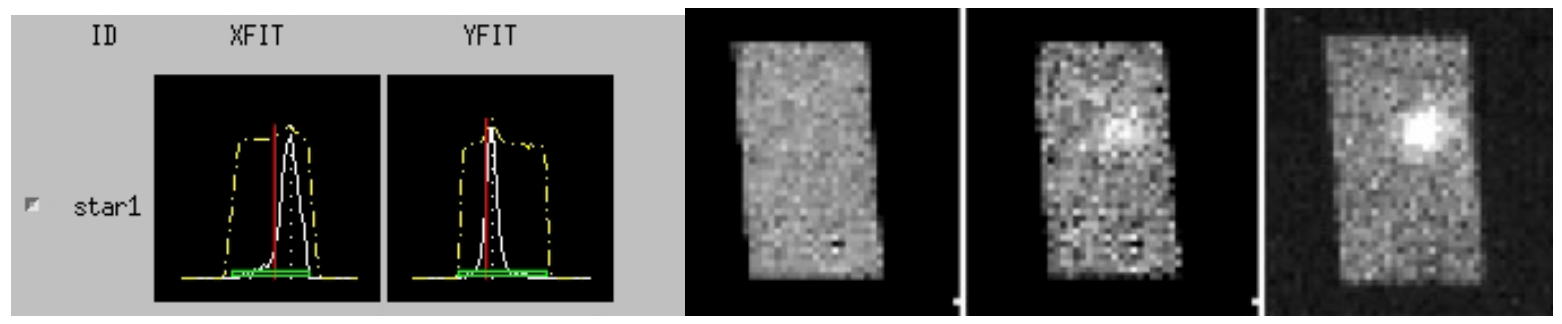

Figure 6. Screen grab from the SAT fine alignment software (left) and the sky, source, and differenced images used for alignment (right). The dashed yellow line is the box plus object profile projected along the rows (x) and columns (y) of the detector. The green box at the bottom indicates where the software identified the box area. The vertical red line marks the desired position of the star. The white line is the object profile after the sky emission was subtracted. The dotted white line is the measured center of the object peak.

\section{MITIGATING ISSUES AND IMPROVING EFFICIENCY}

MOSFIRE's operational performance is exceptionally stable, reliable, and predictable. By the end of the first observing semester (2013A), we had identified all of the common issues that resulted in procedural efficiency losses and a 4-6\% time loss due to faults (see Figure 1). The time lost due to faults in 2013A was below the observatory average by a couple of percent, which reinforced our belief that MOSFIRE was a remarkably well-constructed instrument. The primary issues we did uncover involved CSU motion, detector server image write timeouts, and global keyword server faults. To mitigate these issues and improve observing efficiencies, we made procedural changes and software modifications. In the subsections below, we describe these lessons learned that have led to an increase of more than $10 \%$ in science time in over four years as faults decreased and on-sky science time increased (see Figure 1), and perhaps just as importantly, the modifications resulted in a more pleasant observing experience for MOSFIRE observers.

\subsection{CSU Fatal Errors}

During commissioning and the first observing semester in 2012, the most glaring problem was what became known as a "CSU Fatal Error." This error occurs when the CSU bars are in motion. When the CSU system senses a fault, the electronics power down to protect the hardware from possible damage and the status GUIs warn users that a fatal error occurred. To recover from this fault, we had to initialize the CSU, requiring about 90 minutes to complete.

Frequently, this error occurred during afternoon setup and calibrations, but the error also occurred on sky, resulting in significant time lost. We learned that the frequency was higher in the afternoon for two reasons. First, observers would send a sequence of CSU moves coupled with arcs and flat exposures in relatively short intervals $(<20$ minutes $)$ as part of the calibration script sequencing. Thus, more mask configurations were completed in the few hours before sunset than during the same time at the night. Second, calibrations were acquired with MOSFIRE rotated such that the CSU bars would move parallel to the horizon (rotator positions of 0 or 180 degrees). Although, we expected that this mechanism orientation was the most favorable for bar movement because the motion was orthogonal to the gravity vector, it became clear that the least favorable rotator angles for configuring the CSU were 0 and 180 degrees (i.e. horizontal motion) and that all other rotator angles did not experience the so-called fatal errors (see Figure 7).

Currently, there are no error codes that may be used to track down what is causing the power-down behavior, so we have yet to identify a fix. Instead, we have taken steps to speed-up the recovery process. New software was created to take advantage of the CSU's last known bar state. Following an error, we acquire an image of the current configuration, and then use an edge detection algorithm to pinpoint the bar positions. We then update the state file using our measured bar positions, and force the CSU to read this state file after the controller is power cycled to recover from the fatal error shut down. The measured positions are not accurate enough to precisely configure a mask, but they are accurate enough to move the bars safely back to the open mask configuration. The open mask puts the bars very close to the bar home positions, and homing the CSU from an open mask configuration takes only 25 minutes, saving roughly 60 minutes compared to the full initialization sequence. 


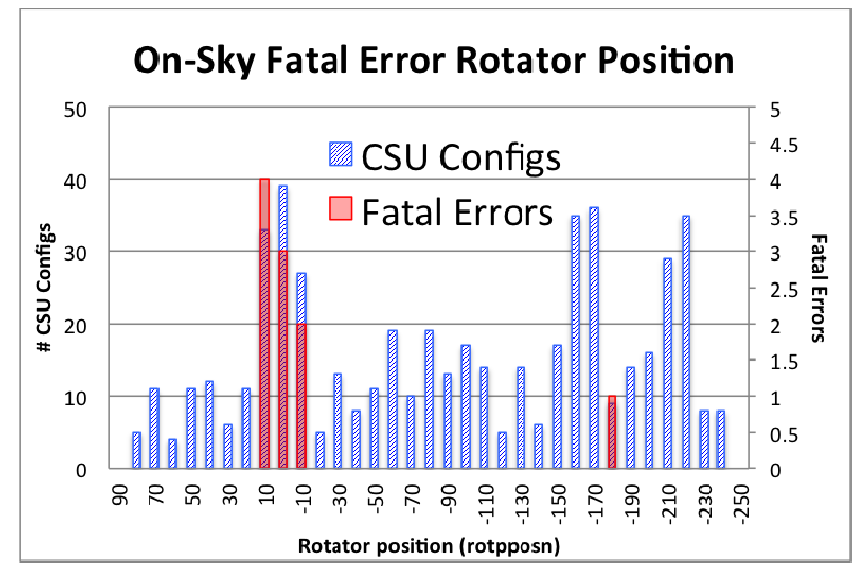

Figure 7. The number of on-sky mask configurations completed during the $2012 \mathrm{~B}$ observing semester relative to the instrument rotator angle (blue). Rotator angles are10 degree bins. Overlaid in red are the number of CSU Fatal Errors.

Of course, the best strategy is to avoid the fatal error shut down completely. Figure 8 shows that we have almost completely eliminated this time loser simply by not configuring the CSU when the rotation of the instrument is near 0 or 180 degrees. Our initial approach was to educate the observing community, as well as our telescope operators, to not reconfigure the CSU at unfavorable drive angles. Every night, versions of the white board photo seen in Figure 8 appeared throughout 2013 as a reminder. The next step was to update the software with a pop-up warning if the drive angle was within 10 degrees of 0 or 180 . The warning prompted observers and telescope operators to rotate the instrument away from the bad drive angle, configure the CSU, and then return to the desired rotator angle needed for the science observations. This tactic nearly eliminated lost time on sky caused by the CSU fatal errors.

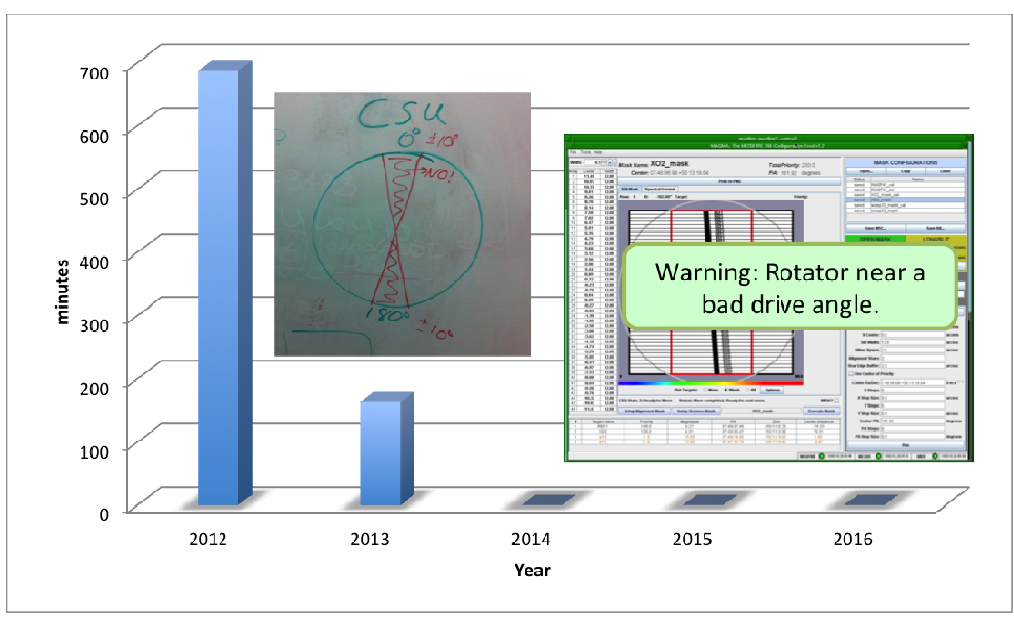

Figure 8. The amount of time lost on-sky due to CSU Fatal Errors over the last five years. The white board drawing photo and the software screen grab are two steps taken to avoid the error.

However, there are two elements to the avoidance strategy that are not ideal. First is that the CSU should not be reconfigured when the rotator is slewing, which reduces observing efficiency. Second, we cannot avoid reconfiguring the $\mathrm{CSU}$ at a potentially bad angle when transitioning from an alignment mask to a science configuration. If we rotated and reconfigured for science, we would lose the on-sky alignment. Because we are usually re-assigning a handful of alignment boxes to science slits, we accept the risks and configure at a bad rotator angle. If bars do fail to redeploy, initializing and reconfiguring just five pairs of bars is much faster than a full CSU initialization sequence. In this situation, we treat recovery just like the case of a bar being out of position (next section).

\subsection{Bars are not at the desired positions}

Figure 5 shows the number of times specific bars were not at the desired location and illustrates that for any mask configuration a specific bar may be out of position every 1 out of 4,000 moves. At this rate, observers can expect to find 
one of the bars out of position roughly once per month. Currently, there is no real-time software that confirms bar positions. Instead, observers are trained to inspect both mask images and spectroscopic observations to detect a poorly positioned bar. Figure 9 provides a sample of what observers are trained to look for during the night.

One of the most helpful and simple additions to the software was to overlay the bar positions in imaging mode. Imaging mode is used for every multi-object slitmask during mask alignment. These alignment images are used not only to fine tune the position of the telescope and rotator to the science field, but are also (hopefully) reviewed by observers. With the bar image overlays, observers note any difference between the green dots marking the locations of the bar edges and the illuminated regions of the detector. Figure 9a, shows a magnified area of an image where bars 87, 88, 89, 91, and 92 are in position while bar 90 did not make it to the desired location. Figure $9 \mathrm{~b}$ shows an instance where the slit width is correct but slit pairs are offset from the desired slit center. In the closed case (Figure 9c), we sometimes detect a sliver of light as can be seen in the upper left of Figure 9c, but often there is no light detected.
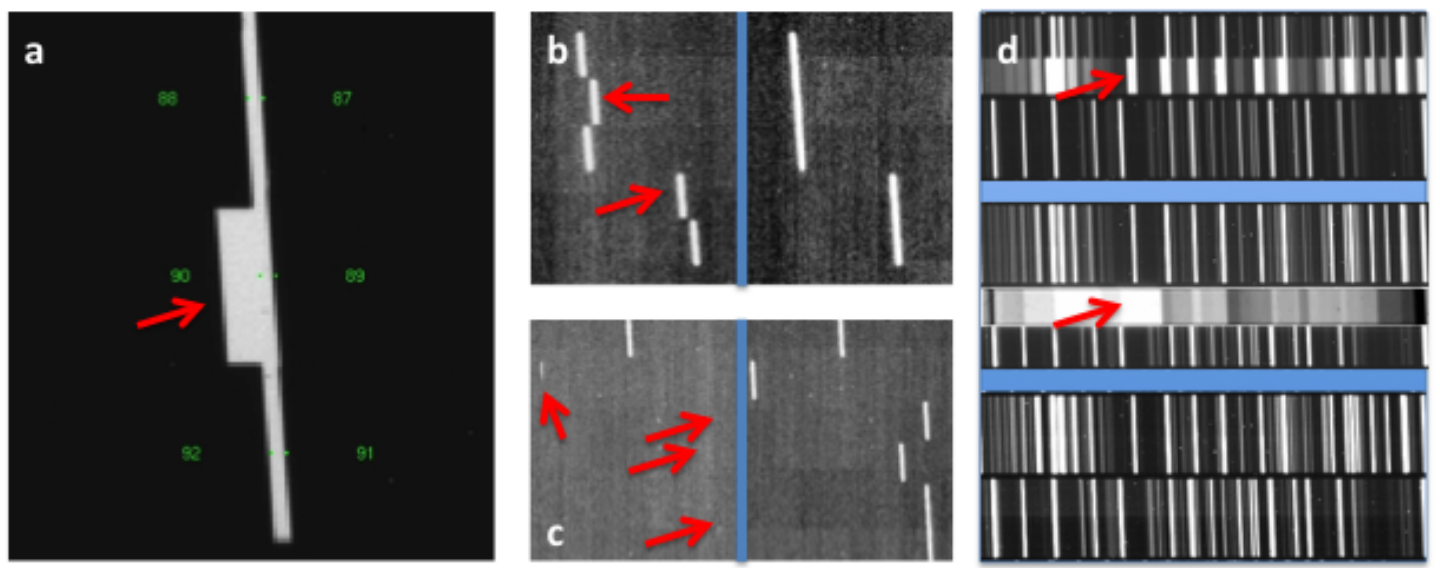

Figure 9. Sample images of bars being out of position: extra wide (a), offset (b), closed (c), extra wide spectroscopic lines (d). Red arrows indicate where the bars are intended to be. For image sets (b) and (c), the right hand side shows the intended configuration, while the desired configuration for (d) is at the bottom. Image (a) is a zoom of a screen shot of the real-time display that overlays labels (green) of the expected positions of the bars.

Figure $9 \mathrm{~d}$ shows a sequence of three images where bars were out of position, and detection was noticed by the appearance of extra wide sky lines in the spectra. After fine alignment of the mask, a bar pair that was used for an alignment box was redeployed to a science location. After bar pairs are redeployed in this manner, we do not re-image the field to determine if the movement was successful. Instead, we examine the sky emission lines in the first few spectroscopic data frames. What is interesting about this particular sequence is that the top image shows the left bar, as indicated by the arrow, out of position. The slit is roughly 4 " wide instead of 0.7 " based on the width of the sky line. We then initialized the bar and sent it back to the desired location. When it went to the desired location, the right hand bar in the slit pair below was caught by the motion and was opened much wider $\left(\sim 15^{\prime \prime}\right)$ as seen in the middle panel of Figure $9 \mathrm{~d}$. We then initialized the second bar, and sent it to the desired location without any further issues (bottom panel).

Based on the visual clues in Figure 9, we suggest some possible scenarios for poor positioning. The first is that the bars skip steps as the ratchets and brakes are cycling through the movement sequence. Missed steps in the coarse or fine positioning may result in a slit pair being wider when a bar does not move closer to its partner, or a closed off slit when a properly positioned slit moves close to its partner that has not moved far enough out of the way. An additional possibility is that sometimes bars drag their neighbors to new positions.

To minimize the number of times bars may be out of position, we initialize the bars at the start of an observing run, and in our afternoon startup procedure, we create a longslit mask to check that bars are at the correct location. When bars are found to be out of position, we have two recovery methods. The first is to move the bar close to the open position and then initialize. This two-step process saves time by moving close to the open position with speeds used for coarse steps instead of searching for the home using only fine motions. This method is applicable for the out-of-position cases illustrated in Figure 9 with the exception of the bar featured in the middle of figure 9d. For that bar, the offset is more than $5^{\prime \prime}$ away and moving it close to the open position will result in an end of limit travel and a fatal error. Instead, we simply initialize from the current position and resend the desired location of the bar. For either process, the recovery time is less than 3 minutes per bar, and as of April 2016, we have only lost 220 minutes to this issue. 


\subsection{CSU electronics thermal dependency}

Until Dec 2015, the CSU electronics needed to be kept warmer than $13 \mathrm{C}$ in order to operate correctly. This thermal dependency was discovered during commissioning when the electronics, which are located in a thermally stabilized cabinet, experienced temperatures in the telescope dome that were colder than lab conditions. Below $13 \mathrm{C}$, the CSU electronics would transmit an amplifier error, and the CSU could not be moved until the electronics were warmed and the impacted bar was initialized. Of course, because all of the MOSFIRE electronics were located in a single cabinet, all of the electronics were subjected to the same heating. Instead of using the highest cooling capacity as intended, it became necessary to throttle back the glycol cooling flow to stop the CSU electronics from getting too cold. Complicating this situation is that the glycol coolant temperature follows the ambient dome temperature, and so we conservatively throttled back the glycol flow to $2 \mathrm{~g} / \mathrm{min}$ such that the internal cabinet temperatures, as measured by sensors in the back of the cabinet, would vary between $18 \mathrm{C}$ and $30 \mathrm{C}$ annually. Although this action helped CSU operations, we experienced over-heating of components associated with the detector system, guiding system, and CSU controller. On very warm nights, the electronics cabinet temperature may exceed the over-temperature threshold of $35 \mathrm{C}$, resulting in a power shutdown of the entire electronics cabinet. All these problems resulted in some lost time. In addition, setting the glycol flow at the telescope was a manual procedure, and a few times, this step was missed, resulting in either a too hot or too cool state.

Thermal sensitivity of the CSU electronics manifests itself in the amplifier circuits on the MACU daughter boards that have two channels for actuating either the brakes or ratchets for two bars. By thermal testing with freeze spray and a power resistor, we narrowed the thermal dependency to a differencing circuit, and through a process of elimination, found that we fixed the thermal dependency by increasing one of the input resistances to the differencing IC by roughly $10 \%$. There were 29 channels that exhibited a thermal dependence, and although there were some boards with two sensitive channels, most boards exhibited a problem with only one channel. Figure 10 shows the amplifier boards being tested with a power resistor in 2013. With these modifications in place, the CSU electronics now operate at 2-3 C, which was the coolest temperature expected for normal use.

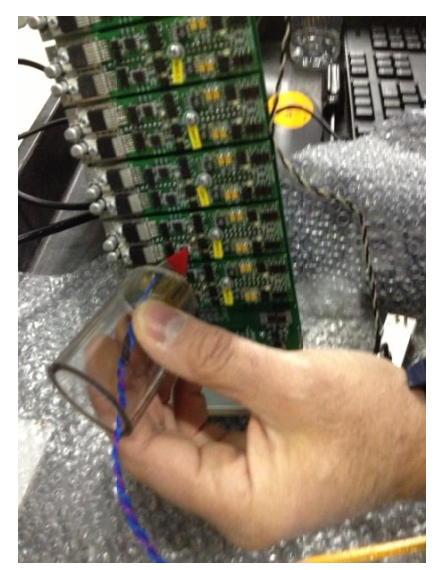

Figure 10. Image of the amplifier board being tested with a power resistor that was used to heat individual components. Board components were individually heated and cooled to isolate where the thermal dependency was located.

\subsection{Detector Server Errors}

MOSFIRE uses a $2 \mathrm{Kx} 2 \mathrm{~K}$ H2-RG HgCdTe detector from Teledyne Imaging Sensors that is readout and clocked using the early version of Teledyne's SIDECAR ASIC technology. The ASIC is packaged separately and is located near the detector inside the vacuum vessel wall. The ASIC is then connected to the JADE2 board that is an interface between the ASIC bus and USB 2.0. The JADE2 is located outside the dewar, and a USB cable connects to an Augmentix computer equipped with a USB driver and solid state disk. The computer runs Windows XP under which the detector control software was developed. This Windows machine is the detector server, and it is located in the MOSFIRE electronics cabinet that must rotate and move with the telescope as the telescope slews in both elevation and azimuth. In order to properly boot the system when powered up, the JADE2 is powered up 30s before the Augmentix reboots and the detector server starts running.

Overall, the system has performed well. There are two small problems associated with the detector server system. The first, and the one that was solved, is that on occasion images would not write to disk in a timely manner. The delay in 
writing data to disk will cause the readout software to timeout and abort an exposure sequence. When constructing a FITS data product, a temporary file call "filename.fits.writing" is created and then later moved to filename.fits. Our investigations indicated that there is a TCP Disconnect that happens from the SidecarServer.exe (random port) to SidecarApp.exe supplied with the system that delayed a file move. When this disconnects, some recoveries took longer than 60 seconds and resulted in a time out.

We have not solved this issue, but we have applied a band-aid in the form of logging that monitors the SidecarApp.exe and SidecarServer.exe. The logging is very minimal. In addition, we have a second script that "touches" files on the network drives every 30 seconds. By keeping the system active via logging and touching files, the file write times are minimized, and time outs are eliminated.

The second issue is a "read timeout failure" and these are typically rare ( 1 out of $\sim 5,000$ exposures). Our suspicion is that there is a connection issue between the JADE2 and the Augmentix computer. This manifests itself as a FITS file with all zero values and a header with keywords that indicate an abort occurred. Because this happens so rarely, the lost time is less than 3 minutes or less, and the failure does not interrupt a sequence of exposures, we have not investigated this issue any further.

\subsection{Slit Drift}

One enormous benefit for MOSFIRE has been feedback from the observing community, who has remained invested in the success of the instrument. The community's careful analysis of MOSFIRE spectra revealed that objects would sometimes drift parallel to the slit length over a two hour observing sequence. Although initially observed as a drift along the slit, careful analysis of the target signal by Dr. Steidel revealed that the stars were also drifting perpendicular to the slit. The community responded by including a bright star in their spectroscopic mask designs so that they could monitor the drift and correct for it during data reduction. After 2.5 years of observing, the MOSDEF and KBSS science teams provided measured drifts over multiple nights, and from those measurements, we developed an empirical model to correct for the observed drift.

Our model has two components. First, differential atmospheric refraction (DAR) contributes about half the observed drift magnitude when observing in the K band. DAR is present because the guider's calculated central wavelength is a constant $872 \mathrm{~nm}$, while the science data are acquired in the YJHK passbands. The second drift component is attributed to mechanical differential flexure between the guider camera and the MOSFIRE detector.

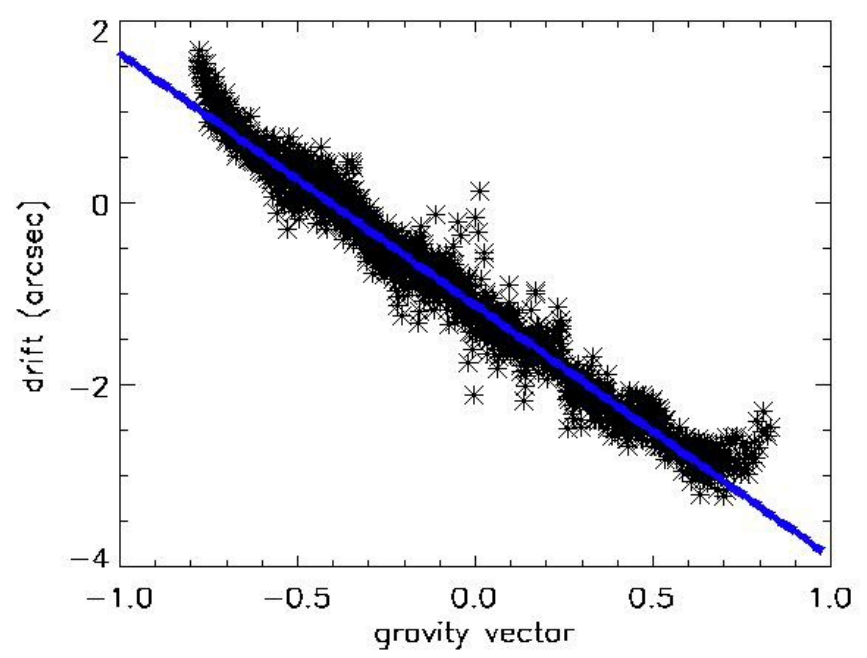

Figure 11. Slit drift with DAR removed. The drift along the slit is relatively linear when plotted against the gravity vector that is a function of both telescope elevation and the instrument rotation. The gravity vector of one implies the vector is parallel to the slit length.

To correct for the mechanical component of the drift, software was added to the observatory guiding system. Because of this effort, the hooks for mechanical flexure correction are now available for all Keck instruments. For DAR correction, MOSFIRE needed to communicate to the telescope what science filter is in use during an observations. Keck staff 
modified GUIs and scripts to monitor the filter changes and update telescope central wavelength guiding parameters. As the telescope elevation and instrument rotation changes to track the sky, the software sends a differential correction relative to the initial elevation and instrument rotation for the observing sequence.

\section{DATA PROCESSING}

The MOSFIRE data reduction pipeline (DRP), developed by the MOSFIRE team under the leadership of N. Konidaris and C. Steidel, has contributed tremendously to the scientific success of the instrument: it has enabled the user community to quickly navigate the intricacies of multi-object infrared spectroscopy and deliver science quality results in refereed publications. Written in Python, the pipeline is able to handle flat fielding, wavelength calibration, and pair subtraction. The end products are 2-D data arrays with the spectra both spatially and spectrally rectified. The pipeline was initially supported and maintained by the MOSFIRE team under the continued leadership of N. Konidaris.

The community warmly supported the MOSFIRE pipeline, rigorously tested the code, and above all else, drove development in new directions by challenging the reduction routines with data sets acquired using new acquisition strategies that took advantage of MOSFIRE's enormous flexibility. Because of this widespread support and drive for further improvements, the observatory decided to invest significant effort in a new initiative to take ownership of the pipeline, centralizing the distribution, provide the user support, and developing new features in partnership and in collaboration with existing MOSFIRE members. The initiative began a new direction for Keck Observatory that, until MOSFIRE, supported a pipeline only if it was required for real-time use.

In 2015, the Observatory produced a new version of the pipeline under the leadership of L. Rizzi, with support for new observing modes, an easier installation procedure and an optionally less interactive interface in particular for wavelength calibration and file management. We serve the code using a publicly available github repository at https://keckdatareductionpipelines.github.io/MosfireDRP/. Because the code is distributed using git, the GitHub was a natural destination for hosting the code, and with it we can:

- Respond to observer requests and concerns using a built-in ticket submission and tracking system

- Merge contributed code from both the community and observatory staff

- Track statistics on code usage and distribution

In the released version, the pipeline is able to autonomously reduce most of the MOSFIRE observations and because it is so robust, the MOSFIRE DRP is planned as an addition to the Keck Observatory Archive (KOA). KOA will eventually serve not just raw MOSFIRE data but also the products from the automated reduction routines. In preparation for KOA and to further improve the code for the MOSFIRE community, new features are being added. We are breaking the dependence on IRAF and a specific Python distribution for easier installation and maintenance. In addition, we hope to provide observers with more data products by adding spectral extraction. Although inclusion into KOA will occur at a later date, the next release of new features to the community is due in the Fall 2016.

\section{FUTURE IMPROVEMENTS}

We continue to seek improvements with MOSFIRE to further increase the on-sky time spent collecting science. There is a point where there may be diminishing returns for our efforts, but we think for MOSFIRE there are still gains to be made. Below we suggest what to pursue in the future to improve the observing experience for our MOSFIRE community.

Although our avoidance strategy has worked remarkably well when configuring the CSU, we would like to characterize as much as we can the bar motions that result in fatal errors. As a metric for why this is important, we can examine the average time required to align a multi-object slitmask, and for our three instruments, those durations are 4.5, 9, and 12 minutes for DEIMOS, LRIS, and MOSFIRE, respectively. The alignment times represent the time between the start of a slew to the next target and the start of the first science exposure on that target. The error avoidance strategy we employ for MOSFIRE all but eliminates faults, but the strategy decreases observing efficiency.

Because most of the overhead with mask alignment involves waiting for the CSU to move, it is difficult to reduce the overheads in aligning a mask. But if we can eliminate CSU fatal errors, we can save on-sky time by rotating and reconfiguring the CSU simultaneously. Over the past four years, 2,845 multi-object slitmasks were aligned on sky. If, the average telescope slew and setup time is 60s per mask, then over the last four years, MOSFIRE observers have waited 
for the telescope for a total of five nights. Thus, we would like to characterize and better understand how to use the CSU during slews and at arbitrary rotator angles.

The strong desire to obtain full wavelength coverage in $\mathrm{H}$ and $\mathrm{K}$ bands motivated the design and supporting software for the long2pos mask (see section 3). If observers want to acquire standards in both $\mathrm{Y}$ or $\mathrm{J}$ and $\mathrm{H}$ or $\mathrm{K}$, two longslit masks are currently used: long2pos for $\mathrm{HK}$ and a single longslit for YJ observations. Because this requires two different masks to be configured, observers are naturally requesting a three position longslit mask that is suitable for all spectroscopic bands and observing scripts and GUIs to orchestrate the data acquisition sequence. Because the offsets are large, predictive tools are needed to help ensure that a guide star will be available for all three slit positions.

A preview of the guider fields would be especially useful for mask design to help select bright guide stars where they are not always readily available in fields such as Hubble Deep fields. We are planning on adding this functionality to the MAGMA software along with a few minor improvements suggested by the observing community. The community is so fond of MAGMA over other mask design tools that the observatory is exploring how to update the mask design software for our two optical multi-object spectrographs with a look, feel, and functionality that matches MAGMA.

Last, having software to confirm bar positions in real-time, rather than relying entirely on observers to recognize the problems presented in section 4.2 is another area for improvement. New observers cannot easily identify a problem, and we have had instances where out-of-position bars went unnoticed by the observing team and support staff.

\section{MOSFIRE ENABLED SCIENCE}

The astronomical science that is completed using MOSFIRE is extensive and diverse. Many observing teams are taking advantage of MOSFIRE's multiplexing capabilities to gain an understanding of larger populations that are statistically significant via long term surveys. But other observing teams are characterizing single objects by taking advantage of the exceptional sensitivity of MOSFIRE on Keck I. Very briefly we highlight below some of the extraordinary science being completed with MOSFIRE.

- Understanding the epoch of re-ionization: Since commissioning, observers have been using MOSFIRE to look back to when the first stars and galaxies started re-ionization the intergalactic medium, a little over 13 billion years ago. Observing teams are probing this epoch by understanding and characterizing the galaxies observed at redshifts of $7<\mathrm{z}<9$ and discerning the stellar masses and star formation rates. Observations with MOSFIRE have been setting redshift records that include $z=7.5123^{15}, 7.66^{10}, 7.73^{16}$, and $8.68^{17}$, and as of 1 June 2016, MOSFIRE has observed and confirmed four out of the six most distant astronomical objects.

- Galaxy surveys:

- The Keck Baryonic Structure Survey (KBSS), led by Dr. Steidel, surveys galaxies at the peak of galaxy formation $(2<\mathrm{z}<3)$ and explores the relationship between intergalactic baryons and galaxies in the same survey volumes. The extent of the sample and the quality of the spectra have enabled the team to map the distribution and properties of gas neighboring star forming galaxies in their target fields. The team is extending their study using MOSFIRE to survey the fields at JHK bands, and the the analysis of a sample of 251 galaxies in 15 fields was one of the first results published ${ }^{5}$ in MOSFIRES's first two years of operation.

- MOSFIRE's expected increase in sensitivity and survey efficiency enabled the MOSFIRE Deep Evolution Field (MOSDEF) Survey team to canvass $\sim 1500$ galaxies so that they may discern the spectroscopic properties at a critical time during the expected assembly of galaxies and the growth of black holes ${ }^{6}$. Observing in the well-studied HST legacy fields AEGIS, COSMOS, and GOODS-N, the MOSDEF team is characterizing the star formation and galactic nuclear activity in these galaxies as well as constraining their masses, morphologies, and their interactions with baryonic matter. The team has amassed nine refereed publications to date ${ }^{18}$.

- Observations with MOSFIRE are challenging previous thinking on the co-evolution of galaxy and black hole growth. In a few galaxies 11 billion light years away, the black hole masses may have 10 percent of the total mass of the galaxy ${ }^{19}$, well above the typical ratio of 1 percent. The observations suggest that during formation, the black hole grew so quickly that the host galaxy was not able to keep up during early evolutionary phases.

- One of the next steps in exoplanet research, now that a few thousand expolanets are known, is to ascertain the composition of their atmospheres, with the goal of finding signatures that may suggest the planet could support life. To make very sensitive measurements of their atmospheric composition, a handful of observing teams have 
created slitmask with 2-4 wide slits (10 arcsec or more) that isolate the light from a transiting planet star system and 1 to 3 spectrophotometric references. MOSFIRE's stability to precisely hold the slit position and the detector sensitivity are enabling the visiting scientist to differentiate between competing atmospheric models for the transiting planet ${ }^{9,20}$. For the transiting ice giant GJ 3470b, MOSFIRE observations confirm that the atmosphere has a hazy, low-methane atmosphere, or alternatively, it may be extremely metal rich $^{9}$.

One of the results to come from MOSFIRE is presented in Figure 12 showing a correlation of Oxygen and Hydrogen abundances with the star formation rate in the universe. The cloud of dark points represent the nearby galaxies $(\mathrm{z}=0)$ while the blue and red markers indicate galaxies with redshifts of $\mathrm{z} \sim 3.5$ tend to have a higher rate of star formation and therefore the typical conditions for star formation must be different than those in the local universe; the conditions require more ionization or radiation pressure to trigger higher star formation rates (SFR). The second interesting thing is that the tail in the local neighborhood of galaxies $(\mathrm{z} \sim 0)$ has similar SFR to the high redshift galaxies. Because the physics must be the same in these galaxy types, we can study the local galaxies with high SFRs to learn more about the early universe.

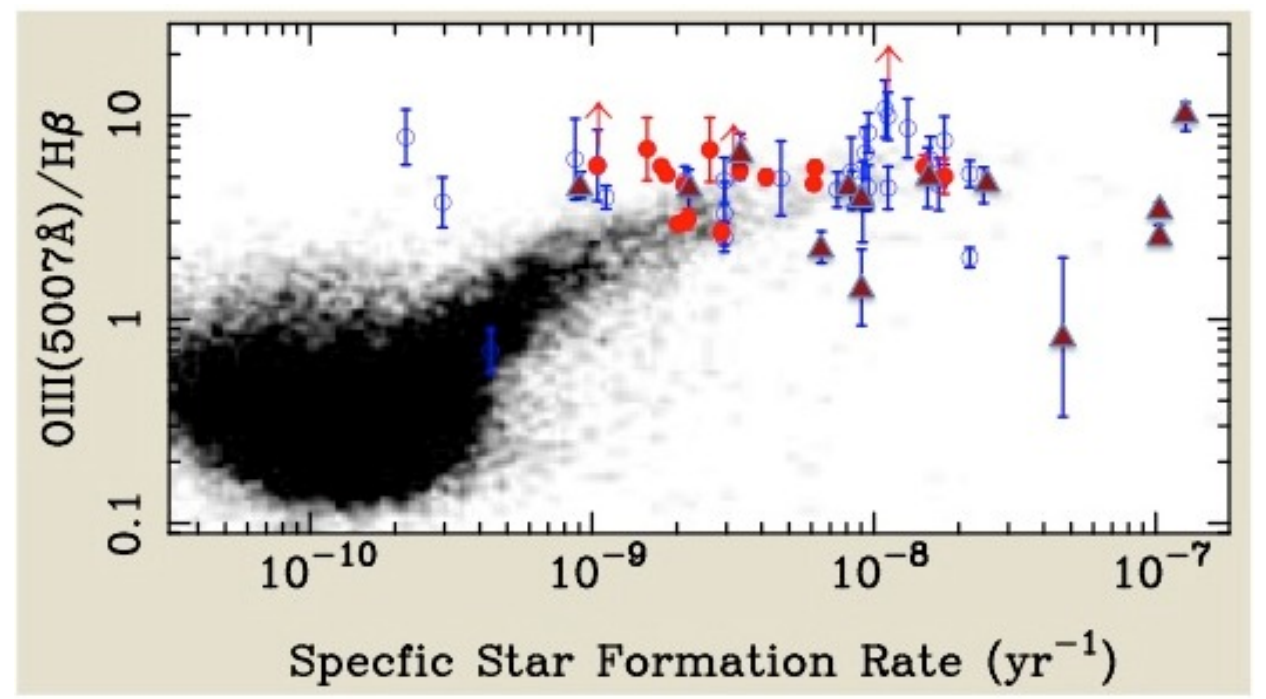

Figure 12. Star formation rate for the local group of galaxies (black) and galaxies at a redshift of Z 3.5 (red and blue). The blue open circles were galaxies observed with SINFONI/ESO while the red circles and triangles were observed on two separate nights with MOSFIRE.

This science result exemplifies MOSFIRE's technological advantages in both sensitivity and multiplexing over other infrared spectrographs. There are three data sets of galaxies at $\mathrm{z} \sim 3.5$ presented in Figure 12. The first observations were acquired using the single object near-infrared spectrograph SINFONI at the European Southern Observatory ${ }^{22}$. The SINFONI observations were acquired one at a time and required 180 hours of on-sky integrations. Using MOSFIRE over two nights ( 20 hours), two observing teams more than doubled the sample size ${ }^{21,23}$. This demonstrates that the multiplexing capabilities provided by the CSU combined with the sensitivity of the detector system, made MOSFIRE at Keck Observatory more efficient by a little more than a factor of nine over other single object infrared spectrographs on $8-10 \mathrm{~m}$ class telescopes.

\section{SUMMARY}

MOSFIRE is taking a lead role in contributing to our understanding of the universe at infrared wavelengths. Because MOSFIRE is operationally friendly, has flexible software, and many issues were mitigated, MOSFIRE is robust and efficiently acquiring images and spectroscopy on a majority of the nights at the W. M. Keck Observatory. Annually, Keck staff in collaboration with MOSFIRE team members and CSEM engineers have improved the instrument either through software or hardware updates that have resulted in an increase of over $10 \%$ of science time.

MOSFIRE's key technological advantage is the multiplexing capability provided by the CSU. This mechanism has shown to be very reliable and predictable. Issues that we have encountered are well behaved and recovery from those 
problems is routine. Both MOSFIRE and CSEM staff have been instrumental in understanding these issues and have been great partners for the four years of MOSFIRE's operation.

\section{REFERENCES}

[1] Spanoudakis, P., Giriens, L., Henein, S., Lisowski, L., O’Hare, A., Onillon, E., Schwab, P. and Theurillat, P., "Configurable slit-mask unit of the Multi-Object Spectrometer for Infrared-Red Exploration for the Keck telescope: Integration and Tests," Proc. SPIE 7018, 7018-19 (2008).

[2] McLean, I. S., Steidel, C. C., Epps, H., Matthews, K., Adkins, S., Konidaris, N., Weber, B., Aliado, T., Brims, G., Canfield, J., Cromer, J., Fucik, J., Kulas, K., Mace, G., Magnone, K., Rodriguez, H., Wang, E., Weiss, J., "Design and development of MOSFIRE, the Multi-Object Spectrometer for Infra-Red Exploration at the Keck Observatory,” Proc. SPIE 7735, 77351E-77351E-12 (2010).

[3] Kulas, K. R., McLean, I. S. and Steidel, C. C., "Performance of the HgCdTe detector for MOSFIRE, an imager and multi-object spectrometer for Keck Observatory," Proc. SPIE 8453, 84531S, 10 pp. (2012).

[4] McLean, I. S., Steidel, C. C., Epps, H. W., Konidaris, N., Matthews, K. Y., Adkins, S., Aliado, T., Brims, G., Canfield, J. M., Cromer, J. L., and 10 coauthors, "MOSFIRE, the multi-object spectrometer for infrared-red exploration at the Keck Observatory," Proc. SPIE 8446, 844600J, 15 pp. (2012).

[5] Steidel, C. C., Rudie, G.C., Strom, A. L., Pettini, M., Reddy, N.A., Shapley, A. E., Trainor, R. F., Erb, D. K., Turner, M. L., Konidaris, N. P., Kulas, K. R., Mace, G., Matthews, K., McLean, I. S., "Strong Nebular Line Ratios in the Spectra of z 2-3 Star Forming Galaxies: First Results from KBSS-MOSFIRE,” ApJ 795, 165, 40 pp. (2014).

[6] Kriek, M., Shapley, A. E., Reddy, N. A., Siana, B., Coil, A. L., Mobasher, B., Freeman, W. R., de Groot, L., Price, S. H., Sanders, R., Shivaei, I., Brammer, G. B., Momcheva, I. G., Skelton, R. E., van Dokkum, P. G., Whitaker, K. E., Aird, J., Azadi, M., Kassis, M., Bullock, J. S., Conroy, C., Davé, R., Keres, D., \& Krumholz, M., "The MOSFIRE Deep Evolution Field (MOSDEF) Survey Rest-frame Optical Spectroscopy for $\sim 1500 \mathrm{H}-$ selected Galaxies at $1.37<\mathrm{Z}<3.8$," ApJS 218, 15 (2015)

[7] Sanders, R. L., Shapley, A. E., Kriek, M., Reddy, N. A., Freeman, W. R., Coil, A. L., Siana, B., Mobasher, B., Shivaei, I., Price, S. H., de Groot, L., "The MOSDEF Survey: Mass, Metallicity, and Star-formation Rate at z 2.3," ApJ 799, 138 (2015).

[8] Wirth, G. D., Trump, J. R., Barro, G., Guo, Y., Koo, D. C., Liu, F., Kassis, M., Lyke, J., Rizzi, L., Campbell, R., Goodrich, R.W., Faber, S. M., "The Team Keck Redshift Survey 2: MOSFIRE Spectroscopy of the GOODS-North Field," ApJ 150, 153 (2015).

[9] Crossfield, I. J. M., Barman, T., Hansen, B. M. S., Howard, A. W., "Warm ice giant GJ 3470b. I. A flat transmission spectrum indicates a hazy, low-methane, and/or metal-rich atmosphere," A\&A 559, 33 (2013).

[10] Song, M., Finkelstein, S. L., Livermore, R. C., Capak, P. L., Dickinson, M., Fontana, A., "Keck/MOSFIRE Spectroscopy of $\mathrm{z}=7-8$ Galaxies: Lyman-alpha Emission from a Galaxy at $\mathrm{z}=7.66$," eprint, asXiv: 1602.02160 .

[11]Faber, S, M., Phillips, A. C., Kibrick, R. I., Alcott, B., et al., "The DEIMOS spectrograph for the Keck II Telescope: integration and testing," Proc. SPIE 4841, 1657 (2003)

[12] Oke, J. B., Cohen, J.G., Carr, M., Cromer, J., Dingzian, A., \& Harris, F.H., "The Keck Low-Resolution Imaging Spectrometer," PASP 107, 375 (1995)

[13] Rockosi, C. et al. "The low-resolution imaging spectrograph red channel CCD upgrade: fully depleted, highresistivity CCDs for Keck," SPIE 7735, 26 (2010)

[14] Kassis, M., Wirth, G. D., Phillips, A. C., \& Steidel, C. C., "The slitmask alignment tool: robust, efficient, and astronomer-friendly software for aligning multi-object slitmasks," Proc. SPIE 8448, 844807-15 (2012)

[15] Finkelstein, S. L., Papovich, C., Dickinson, M., Song, M., Tilvi, V., Koekemoer, A. M., Finkelstein, K. D., Mobasher, B., Ferguson, H. C., Giavalisco, M., Reddy, N., Ashby, M. L. N., Dekel, A., Fazio, G. G., Fontana, A., Grogin, N. A., Huang, J.-S., Kocevski, D., Rafelski, M., Weiner, B. J., Willner, S. P., "A galaxy rapidly forming stars 700 million years after the Big Bang at redshift 7.51," Nature 502, 524 (2013)

[16] Oesch, P. A., van Dokkum, P. G., Illingworth, G. D., Bouwens, R. J., Momcheva, I., Holden, B., RobertsBorsani, G. W., Smit, R., Franx, M., Labbé, I., González, V., Magee, D., "A Spectroscopic Redshift Measurement for a Luminous Lyman Break Galaxy at z=7.730 using Keck/MOSFIRE," AJ 804, 30 (2015). 
[17]Zitrin, A, Labbé, I, Belli, S., Bouwens, R., Ellis, R. S., Roberts-Borsani, G., Stark, D. P., Oesch, P. A., Smit, R., "Lyman-alpha Emission from a Luminous z=8.68 Galaxy: Implications for Galaxies as Tracers of Cosmic Reionization," eprint, arXiv:1507.02679

[18] Coil, A., Kriek, M., Mobasher, B, Reddy, N., Shapley, A., Siana, B., "The MOSFIRE Deep Evolution Field Survey," 1 June 2016, http://mosdef.astro.berkeley.edu

[19] Trakhtenbrot, B., Urry, C. M., Civano, F., Rosario, D. J., Elvis, M., Schawinski, K., Suh, H., Bongiorno, A., Simmons, B. D., "An over-massive black hole in a typical star-forming galaxy, 2 billion years after the Big Bang," Science 349, 168 (2015)

[20] Morris, B., Mandell, A., Angerhausen, D., Kassis, M., Madhusudhan, N, McElwain, M. W., "Exoplanet Transmission Spectroscopy in the Near Infrared with Keck/MOSFIRE,” AAS 225, 257.10 (2015)

[21] Holden, B. P., Oesch, P. A., González, V. G., Illingworth, G. D., Labbé, I., Bouwens, R., Franx, M., van Dokkum, P., Spitler, L. "Rest-frame Optical Emission Lines in z 3.5 Lyman-break-selected Galaxies: The Ubiquity of Unusually High [OIII]/H $\beta$ Ratios at 2 Gyr," ApJ 820, 73 (2016)

[22] Troncoso, P., $\quad$ Maiolino, R., Sommariva, V., Cresci, G., $\quad$ Mannucci, F., $\quad$ Marconi, A., $\quad$ Meneghetti, M., Grazian, A., Cimatti, A., Fontana, A., Nagao, T., Pentericci, L., "Metallicity evolution, metallicity gradients, and gas fractions at $\mathrm{z} \sim 3.4$, , A\&A 563, 58 (2014)

[23] Schenker, M. A., Ellis, R. S., Konidaris, N. P., Stark, D. P., "Contamination of Broadband Photometry by Nebular Emission in High-redshift Galaxies: Investigations with Keck's MOSFIRE Near-infrared Spectrograph,” ApJ 777, 67 (2013) 